(C) The Author(s), 2021. Published by Cambridge University Press on behalf of The Nutrition Society. This is an Open Access article, distributed under the terms of the Creative Commons Attribution licence (https://creativecommons.org/licenses/by/4.0/), which permits unrestricted re-use, distribution, and reproduction in any medium, provided the original work is properly cited.

\title{
Impact of Christian Orthodox Church dietary recommendations on metabolic syndrome risk factors: a scoping review
}

\author{
A. Kokkinopoulou ${ }^{1,2 *}$ (1) and A. Kafatos ${ }^{1}$ \\ ${ }^{1}$ Department of Preventive Medicine and Nutrition Unit, School of Medicine, University of Crete, Heraklion, Greece \\ ${ }^{2}$ Department of Nutritional Sciences and Dietetics, International Hellenic University, Thessaloniki, Greece
}

\section{Abstract}

Fasting has been a practice among followers of different religions for many years. Christian Orthodox Church (COC) fasting is a periodic vegetarian-type diet in which seafood and snails are allowed on most fasting days. The present scoping review aimed to present available data regarding the benefits of COC fasting on metabolic syndrome (MetS) risk factors. Databases were searched for available studies. Twenty publications, with a total of 1226 fasting participants, provided data on the effects on different variables of MetS, including blood pressure, blood lipids and anthropometric measurements. Fasters' diet is characterised by low saturated and trans fat intake, high complex carbohydrate and fibre consumption, due to permissible foods. COC fasting has no deficiency in essential amino acid intake since seafood and snails are allowed on fasting days. Fasters have healthier blood lipid profiles during and after COC fasting periods, and total cholesterol, LDL-cholesterol, systolic blood pressure, body weight and BMI are reduced after a fasting period. Due to restricted or forbidden intake of specific foods during the COC fasting periods, one might expect that fasters have reduced intake of macro- and micronutrients, but as shown in the available literature, there are no deficiencies. Future research on COC fasting is needed in areas not investigated at all, like Mets, before reaching definite conclusions.

Keywords: Religious fasting: Christian Orthodox Church fasting: Metabolic syndrome: Cardiovascular health: Plant-based diets (Received 3 November 2020; revised 10 May 2021; accepted 1 June 2021; accepted manuscript published online 10 June 2021)

\section{Introduction}

Fasting is defined as voluntary abstention from food for varying duration $^{(1)}$, and it has been positively associated with longevity and boosting of human health ${ }^{(2)}$. Religious fasting (RF) is a nutritional model that has various levels of food restrictions as a dietary regimen of most popular religions ${ }^{(3)}$. The possible impact of RF on human health has been the subject of public debate for thousands of years. Over the last two decades, an increasing number of studies have been carried out on RF and its impact on human health. Evidence suggests that RF may have preventive effects on various diseases, such as obesity, cardiovascular diseases (CVD) and type 2 diabetes mellitus (T2DM) ${ }^{(1,2,4)}$.

The Christian Orthodox Church (COC) suggests that individuals who follow the long-term structured fasting type of diet abstain from meat, dairy products and eggs for 180-200 d annually, while their diet is characterised by increased consumption of cereals, legumes, fruits, vegetables, fish and seafood. COC fasting includes three main fasting periods: $40 \mathrm{~d}$ prior to Christmas (from 15 November to 24 December), $48 \mathrm{~d}$ prior to Easter (from Clean Monday to Holy Saturday), $14 \mathrm{~d}$ prior to Assumption (from 1 to 14 August), the fasting period prior to the feast of Holy Apostles (lasting 0-30 d depending on Easter feast), and three other daily feasts (5 January, 29 August, 14 September), as well as every Wednesday and Friday ${ }^{(3,5)}$.
Details about foods to be avoided and the duration of fasting periods can be found in Table 1 .

COC fasting has unique dietary recommendations demonstrating an infrequent interchange from a mixed diet to a vegetarian diet that includes fish and seafood ${ }^{(6)}$. The periodic fasting followed by Orthodox Christians in Greece during all COC fasting periods resembles the traditional Greek diet, with plenty of legumes, vegetables, fruits, nuts, olives, bread, fish and seafood, such as shrimps, octopus and snails ${ }^{(3-5,7,8)}$. Since the results of the Seven Countries Study (SCS), the Mediterranean diet and more specifically the diet of Crete have been identified as a healthy diet associated with CVD protection and promoting longevity ${ }^{(9)}$. The population of Crete of the SCS had the lowest CHD mortality and the highest life expectancy in comparison with all other fifteen cohorts $^{(10)}$. The term Mediterranean diet was generated from the SCS and the diet of Crete in 1960 when $60 \%$ of Greek participants fasted strictly during all fasting periods of the COC ${ }^{(10-12)}$. The longest adult life expectancy and the lowest mortality rates from all chronic diseases seem to be related to the excellent dietary pattern of the population of Crete and the daily intense physical activity ${ }^{(12,13)}$. This lifestyle of the population of Crete in the 1960s resulted in the lowest body weight in comparison with all other fifteen cohorts of the SCS during the 50 years of followup $^{(14)}$. Also, between 1994 and 1997, the European Prospective

* Corresponding author: Anna Kokkinopoulou; email: kokkinopoulouan@gmail.com 
Investigation into Cancer (EPIC) study was undertaken in Greece with 28572 individuals aged 20-86 years participating. After a mean follow-up of 8.5 years, lower overall mortality was found in comparison with that of to the individuals with closer adherence to the traditional Mediterranean diet, ${ }^{(15)}$ and as it was observed, COC fasting was followed by $50 \%$ and $65 \%$ of men and women, respectively, who participated in the Greek cohort of $\mathrm{EPIC}^{(3)}$.

Metabolic syndrome (MetS) has become a public health concern and, as defined by the World Health Organization (WHO), is a cluster of disorders that includes abdominal obesity, insulin resistance, dyslipidaemia and hypertension ${ }^{(16)}$. Different definitions of MetS from several organisations exist, with the most commonly used for health care plans coming from the $\mathrm{WHO}^{(17)}$, the National Cholesterol Education Program Adult Treatment Panel III (NCEP ATP III) ${ }^{(18)}$ and the International Diabetes Federation (IDF) ${ }^{(19)}$. Criteria for clinical diagnosis of the MetS are (i) elevated waist circumference with cut-off points according to population- and country-specific definitions, (ii) elevated triglycerides with cutoff point $\geq 150 \mathrm{mg} / \mathrm{dL}$, (iii) reduced HDL-cholesterol with cutoff points $<50 \mathrm{mg} / \mathrm{dL}$ in females and $<40 \mathrm{mg} / \mathrm{dL}$ in males, (iv) elevated blood pressure with cut-off points $\geq 130 \mathrm{mmHg}$ for systolic and/or $\geq 85 \mathrm{mmHg}$ for diastolic blood pressure and (v) elevated fasting glucose with cut-off point $\geq 100 \mathrm{mg} / \mathrm{dL}^{(20)}$. Prevalence of MetS is increasing rapidly throughout the world, and having it increases the risk of developing CVD twofold and T2DM fivefold ${ }^{(20)}$. The prevalence of MetS in Greece according to the METS-GREECE Multicentre Study is $23.6 \%{ }^{(21)}$, and according to the ATTICA study is $19.8 \%{ }^{(22)}$, with both studies using the NCEP ATP III definition for MetS prevalence.

The NCEP ATP III and the American Heart Association have recommended a diet approach to prevent MetS as part of a multifaceted lifestyle approach to reduce CVD risk ${ }^{(23)}$. Part of this approach is being physically active by following the national guidelines for moderate- and/or high-intensity physical activity, as it decreases chronic disease mortality ${ }^{(24)}$. Among the 1627 participants of the SUN cohort study that were followed up for 6 years, those with the highest adherence to the Mediterranean food pattern had the lowest levels of all risk factors of MetS ${ }^{(25)}$. Similar results were demonstrated by the PREDIMED study, as it was suggested that adherence to a Mediterranean diet had beneficial effects on the reversion of MetS. Among 5801 individuals, aged 55-80 years, with a 4.8 year follow-up, the ones having the highest adherence to the Mediterranean diet had a $56 \%$ lower likelihood of having MetS ${ }^{(26,27)}$. Also, the ATTICA study revealed that, in a population of 2282 individuals over 18 years old who were followed for approximately 8.5 years, those who had higher adherence to the Mediterranean diet reduced their odds of having MetS by $20 \%{ }^{(22)}$.

Overall, there is growing scientific evidence of how the Mediterranean diet affects MetS risk factors, and limited evidence on the association between COC fasting dietary recommendations and MetS. This scoping review aimed to explore the evidence on the association between the COC long-term structured fasting and various aspects of human health and MetS risk factors. To our knowledge, no study has investigated the effects of the long-term fasting recommendation of the COC diet on MetS. Existing gaps in the available literature and future research are also discussed. 


\section{Methods}

This scoping review was conducted to find evidence on the association between COC fasting and MetS risk factors. We aimed to respond to this significant research question in a comprehensive manner via the scoping review method. This research method does not include a risk of bias of the evidence, as it reports evidence from existing studies and identifies gaps in the available literature $^{(28)}$. The scoping review was made in accordance with the Preferred Reporting Items for Systematic reviews and MetaAnalyses (PRISMA) guidelines ${ }^{(29)}$, and the protocol is presented in detail below. PRISMA 2009 Checklist is included as supplementary material.

\section{Literature search}

The authors performed an extensive literature search for published articles from February until March 2020 from the following datasets: PubMed (via MEDLINE), CINAHL, Scopus and Google Scholar. An additional search was performed in June 2020 for new available publications.

To retrieve relevant literature, the search strategy involved the following keywords with any possible combination in the abstracts of the articles: 'religious fast' or 'religious fasting' or 'Christian Orthodox fast' or 'Christian Orthodox Church fasting' or 'Greek Orthodox fast' or 'Greek Orthodox fasting' or 'Greek Orthodox Church fasting' or 'Greek monks' and 'health' or 'obesity' or 'blood lipids' or 'cardiovascular diseases' or 'metabolic syndrome'.

\section{Inclusion and exclusion criteria}

Criteria for the included studies were the following: (i) studies on humans; (ii) studies with anthropometric measurements, such as body weight and waist circumference; (iii) studies with nutrition results including food choices; (iv) studies with biochemical metabolic markers including total cholesterol, HDL-cholesterol, LDL-cholesterol, triacylglycerol, HbA1c and blood pressure levels; (v) studies published in English and Greek language; (vi) studies published from January 1990 to March 2020; and (vii) full-length papers. On the other hand, reviews, editorials, meta-analyses, animal studies, study protocols, abstracts and unpublished results were initially excluded from the review process. Additionally, studies were excluded if focused religion was different than COC and if reported measurements were not health related. No restrictions were made regarding study sample and/or fasting duration.

\section{Data collection and analysis}

After duplicate publications removal, abstracts of full publications were assessed for eligibility in this review, in accordance with the inclusion and/or exclusion criteria. Data extracted from each publication are: name of the first author, publication year, aim of the study, study design, country of study, sample size and characteristics of participants, fasting periods, results concerning anthropometric measurements, arterial blood pressure and haematologic data. No meta-analysis was performed due to heterogeneity of studies and reported outcomes.
Results

\section{Eligible articles}

The initial search of the databases identified 1169 articles, and 35 more articles were found through manual searching. A total of 286 duplicates were removed, leaving 918 articles to be considered for inclusion after title and abstract screening. Fifty-one full publications were reviewed for eligibility, with twenty publications finally included in our scoping review. The process for identifying and selecting papers for this scoping review is in accordance with PRISMA guidelines and presented in the PRISMA flow diagram in Fig. 1. We also used the quality assessment tool as proposed by Hawker and colleagues ${ }^{(30)}$ for the included publications, to screen the studies, which can be found in Appendix 1. Results show that most of the publications (18/20) were defined as high quality and only a few (2/20) defined as medium quality.

In total, twenty publications from eleven unique studies that investigate the relationship between COC fasting and health indices met the eligibility criteria and were finally included in this review. Out of them, two were cross-sectional studies (producing four publications ${ }^{(31-34)}$ ), one was a case-control study (producing five publications ${ }^{(6,35-38)}$ ) and eight were prospective studies (producing eleven publications $\left.{ }^{(39-49)}\right)$.

\section{Overview of the studies and study sample}

Most of the data were collected from Greece, a country where the majority of the people are Christian Orthodox, with study sample coming from different origins of Greece: mainly from Crete and Northern Greece, as two studies were conducted in Crete with seven publications ${ }^{(6,35-38,42,43)}$; five different studies in Northern Greece produced eight articles ${ }^{(31-34,44-46,49)}$; and one study in Peloponnese with one published article ${ }^{(41)}$. Also, two unique studies were conducted in Egypt with two publications $^{(47,48)}$ and one in the USA with two different published $\operatorname{articles}^{(39,40)}$.

In five publications, fasters were people under religious orders, that is, nuns and monks, mainly living in monasteries ${ }^{(41-45)}$; in six, fasters were a mix of nuns, monks, priests and laypeople ${ }^{(6,35-38,46)}$; and in nine, fasters were only laypeople ${ }^{(31-34,39,40,47-49)}$. In nine publications, a control group was included in the analysis, with laypeople being non-fasters ${ }^{(6,31-33,35-38,48)}$.

In all studies, participants did not suffer from chronic illnesses and/or received medication and/or supplements. The exact number of recruited participants in all available publications cannot be summed, as there is potential overlap of cases and controls in publications conducted by the same research group ${ }^{(6,31-33,35-40,42,43,45,46)}$. Sample size in each publication varies from 1 person $^{(42)}$ to 609 participants $^{(34)}$, and target age group varies from 18 to 84 years old in all including articles with the exception of one article focusing on a paediatric population aged $5-15 \cdot 5$ years old ${ }^{(34)}$.

Methodology and main outcomes of all COC fasting publications included in this review are displayed in Table 2. 


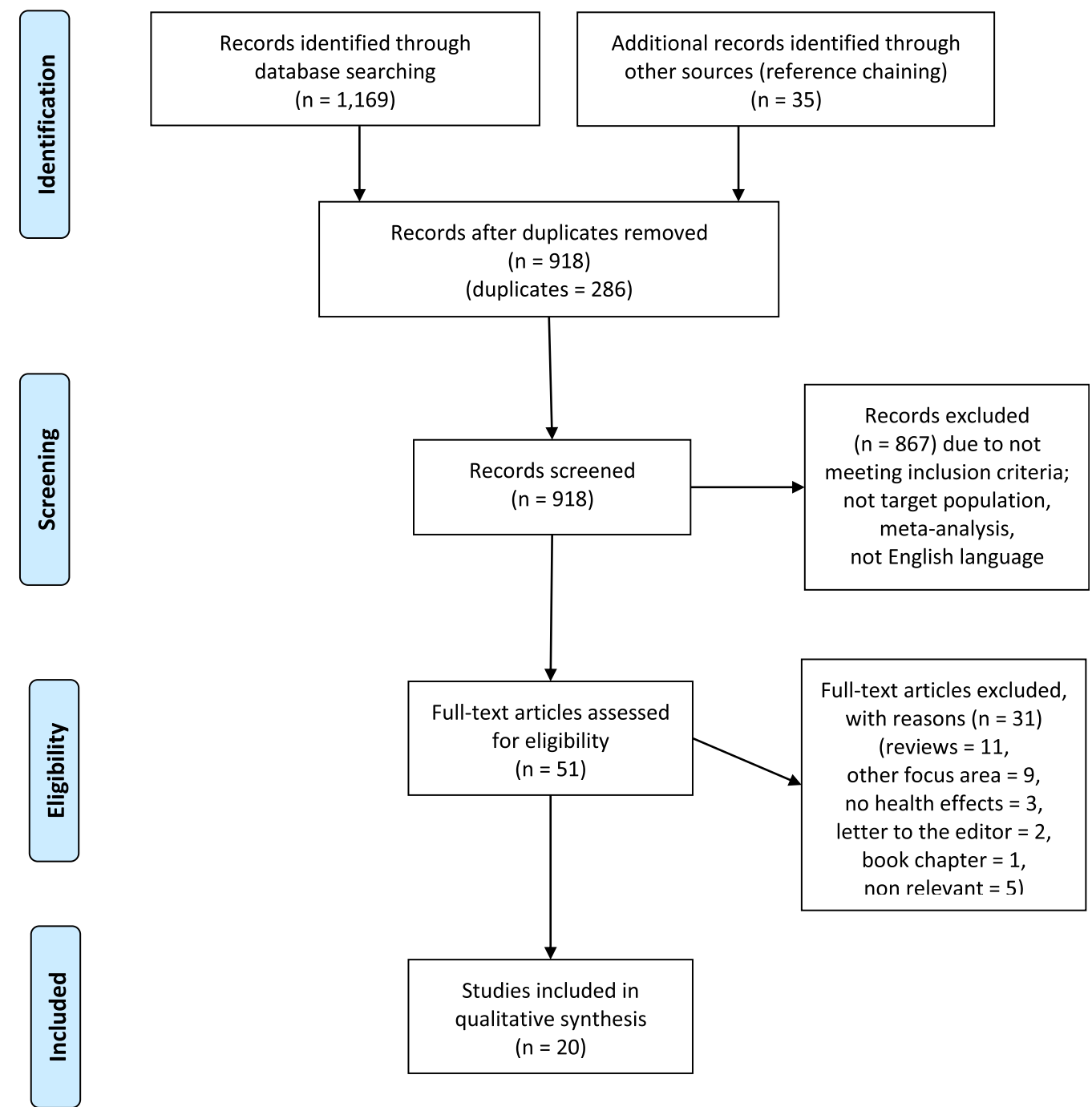

Fig. 1. Flow chart diagram according to the PRISMA guideline.

\section{Frequency of COC fasting practices}

Publications included in the analysis vary in duration of examined COC fasting periods between $1 \mathrm{~d}^{(45,46)}, 7 \mathrm{~d}^{(42,43)}$ and longer fasting periods ${ }^{(6,35-44,47-49)}$. Data on longer COC fasting periods are available from five publications examining the effects of Easter fasting period ( $48 \mathrm{~d})^{(39,40,44,48,49)}$, four examining the effects of Christmas fasting period (40-43 d depending on the country) ${ }^{(36,38,41,47)}$ and three focused on three main COC fasting periods, that is, Easter,

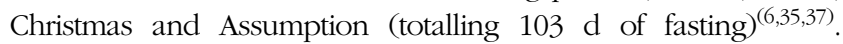
Lastly, five did not focus on specific COC fasting periods, but rather on the effect of adherence to the COC fasting principles ${ }^{(31-34)}$.

Some of them reported the duration of the COC fasting adherence. Fasters in one publication had been observing fast for 14 years $^{(32)}$, one reported a mean of $24 \pm 10 \cdot 4$ years adherence to the COC fasting rituals ${ }^{(43)}$, in others a mean of $20 \pm 14$ years was mentioned ${ }^{(6,35-38)}$, in one a mean of 31 years adherence was reported $^{(31)}$ and in another one a mean of 32 years average fasting time was reported ${ }^{(33)}$. In the case of participants being under religious orders, one study mentioned $12 \cdot 5 \pm 8 \cdot 2$ years in monastic life ${ }^{(43)}$, and in the case of the Athonian monks,
6 months adherence to COC fasting was sufficient to be included in the study ${ }^{(45,46)}$.

Different dietary assessment methods were used in the publications, and all results are comparable since the methods used are validated. One 24-h dietary recall in combination with a 3 -d weighed food record was used in five publications ${ }^{(6,35-38)}$, three 24-h dietary recalls and a food frequency questionnaire in three publications ${ }^{(5,32,33)}, 7-\mathrm{d}$ weighed food records in three publications ${ }^{(41-43)}, 7-d$ weighed food records in combination with a food frequency questionnaire in two publications ${ }^{(39,40)}$, 3-d weighed food records in one publication ${ }^{(34)}, 2-\mathrm{d}$ weighed food records in two publications ${ }^{(45,46)}$ and a food frequency questionnaire in one publication ${ }^{(47)}$, while three publications did not focus on dietary intake at all ${ }^{(44,48,49)}$. Data regarding the dietary assessment method used in each publication and main nutritional outcomes are displayed in Table 3.

\section{Blood pressure levels}

A few studies have investigated the effect of COC fasting regimes on blood pressure, with mixed results. 
Table 2. Details of COC fasting publications included in the review

\begin{tabular}{|c|c|c|c|c|c|c|c|c|}
\hline$a / a$ & $\begin{array}{l}\text { Author(s), year, } \\
\text { country }\end{array}$ & Study design & Sample size (n) & $\begin{array}{l}\text { Target age } \\
\text { groups } \\
\text { (years) }\end{array}$ & Fasting period & $\begin{array}{l}\text { Anthropometric mea- } \\
\text { surements }\end{array}$ & Arterial blood pressure & Haematologic data \\
\hline 1 & $\begin{array}{l}\text { Rodopaios et al., } \\
\text { 2020, Greece }\end{array}$ & $\begin{array}{l}\text { Cross-sec- } \\
\text { tional study }\end{array}$ & $\begin{array}{l}100 \mathrm{~F} \\
100 \mathrm{NF}\end{array}$ & $>50$ & NFP & $\begin{array}{l}4 \% \text { of } \mathrm{F} \text { had } \\
\text { osteoporosis com- } \\
\text { pared with } 14 \% \text { of } \\
\mathrm{NF}\end{array}$ & NA & NA \\
\hline 2 & $\begin{array}{l}\text { Rodopaios et al., } \\
\text { 2020, Greece }\end{array}$ & $\begin{array}{l}\text { Cross-sec- } \\
\quad \text { tional study }\end{array}$ & $\begin{array}{l}100 \mathrm{~F} \\
100 \mathrm{NF}\end{array}$ & $18-35$ & NFP & $\begin{array}{l}\text { BW and BMI did not } \\
\text { differ between } \\
\text { groups. } \\
\text { Bone mineral density } \\
\text { and bone mineral } \\
\text { content did not dif- } \\
\text { fer between } \\
\text { groups }\end{array}$ & $\begin{array}{l}\text { SBP and DBP did not dif- } \\
\text { fer between groups }\end{array}$ & $\begin{array}{l}\text { Serum calcium and vitamin } \mathrm{D} \text { did not differ } \\
\text { between groups, while urea was higher in NF }\end{array}$ \\
\hline 3 & $\begin{array}{l}\text { Bethancourt et al., } \\
2019, \text { USA }^{(40)}\end{array}$ & $\begin{array}{l}\text { Prospective } \\
\text { study }\end{array}$ & $99 \mathrm{~F}$ & $19-73$ & $48 \mathrm{~d}$, Easter FP & $\begin{array}{l}\mathrm{F} \text { reduced } \mathrm{BW} \text { after a } \\
\mathrm{FP}\end{array}$ & NA & $\begin{array}{l}\text { F had lowered total cholesterol and LDL-choles- } \\
\text { terol after a FP }\end{array}$ \\
\hline 4 & $\begin{array}{l}\text { Bethancourt et al., } \\
\text { 2019b, USA }\end{array}$ & $\begin{array}{l}\text { Prospective } \\
\text { study }\end{array}$ & $99 \mathrm{~F}$ & $19-73$ & $48 \mathrm{~d}$, Easter FP & NA & NA & NA \\
\hline 5 & $\begin{array}{l}\text { Rodopaios et al., } \\
\text { 2019, Greece } \\
(5)\end{array}$ & $\begin{array}{l}\text { Cross-sec- } \\
\text { tional study }\end{array}$ & $\begin{array}{l}100 \mathrm{~F} \\
100 \mathrm{NF}\end{array}$ & $50-78$ & NFP & $\begin{array}{l}\text { BW and BMI did not } \\
\text { differ between } \\
\text { groups. } \\
\text { Bone mineral density } \\
\text { and bone mineral } \\
\text { content did not dif- } \\
\text { fer between } \\
\text { groups }\end{array}$ & $\begin{array}{l}\text { F had lower DBP, but } \\
\text { SBP did not differ } \\
\text { between groups }\end{array}$ & $\begin{array}{l}\text { Serum calcium, vitamin } \mathrm{D} \text { and urea did not differ } \\
\text { between groups }\end{array}$ \\
\hline 6 & $\begin{array}{l}\text { Karras et al., } \\
2019, \text { Greece }^{(46)}\end{array}$ & $\begin{array}{l}\text { Prospective } \\
\text { study }\end{array}$ & $100 \mathrm{~F}$ (100 male) & $20-45$ & $\begin{array}{l}1 \text { fasting (Easter) } \\
\text { and } 1 \text { non-fast- } \\
\text { ing day }\end{array}$ & $\begin{array}{l}\text { Athonian monks had } \\
\text { lower BMI, BF } \\
\text { mass, WC, VF } \\
\text { mass }\end{array}$ & NA & $\begin{array}{l}\text { All F had normal total cholesterol and LDL-cho- } \\
\text { lesterol levels, and low HDL-cholesterol lev- } \\
\text { els. } \\
\text { Athonian monks had better fasting insulin con- } \\
\text { centrations and HOMA-IR values, PTH was } \\
\text { higher, calcium level was normal and vitamin } \\
\text { D level was low }\end{array}$ \\
\hline 7 & $\begin{array}{l}\text { El-Sayed et al., } \\
\text { 2018, Egypt }{ }^{48)}\end{array}$ & $\begin{array}{l}\text { Prospective } \\
\text { study }\end{array}$ & $\begin{array}{l}49 \mathrm{~F}(25 \text { female, } \\
24 \text { male) } \\
48 \mathrm{NF}(22 \text { female, } \\
26 \text { male) }\end{array}$ & $22-84$ & $48 \mathrm{~d}$, Easter FP & $\begin{array}{l}\text { F with and without } \\
\text { T2DM lowered } \\
\text { their BMI and WC } \\
\text { after a FP }\end{array}$ & $\begin{array}{l}\text { F with and without T2DM } \\
\text { lowered their SBP, F } \\
\text { with hypertension low- } \\
\text { ered both SBP and } \\
\text { DBP after a FP.ç }\end{array}$ & $\begin{array}{l}\text { F with T2DM had no changes in fasting blood } \\
\text { glucose, lowered } \mathrm{HbA1c} \text { and triacylglycerol } \\
\text { levels, while no changes in cholesterol, LDL } \\
\text { and HDL were found. } \\
\text { F without T2DM increased fasting blood glucose } \\
\text { levels after a FP }\end{array}$ \\
\hline 8 & $\begin{array}{l}\text { Makedou et al., } \\
\text { 2018, Greece }\end{array}$ & $\begin{array}{l}\text { Prospective } \\
\text { study }\end{array}$ & $35 \mathrm{~F}$ & $19-66$ & $48 \mathrm{~d}$, Easter FP & NA & NA & $\begin{array}{l}\text { F decreased WBC, granulocytes, haemoglobin, } \\
\text { RBC, MCV, platelets, INR, aPTT and FVII } \\
\text { activity, while lymphocytes and TAC level } \\
\text { were increased after a FP }\end{array}$ \\
\hline 9 & $\begin{array}{l}\text { Elshorbagy et al., } \\
2017, \text { Egypt }^{(47)}\end{array}$ & $\begin{array}{l}\text { Prospective } \\
\text { study }\end{array}$ & $\begin{array}{l}36 \mathrm{~F} \text { ( } 24 \text { female, } \\
12 \text { male) }\end{array}$ & 29-32 & $\begin{array}{l}43 \mathrm{~d} \text {, before } \\
\text { Christmas FP }\end{array}$ & $\begin{array}{l}\text { BMI was decreased } \\
\text { after the FP, while } \\
\text { \%BF remained the } \\
\text { same after a FP }\end{array}$ & NA & $\begin{array}{l}\text { F decreased cholesterol levels, while glucose } \\
\text { and insulin remained the same after a FP. } \\
\text { Leucine, isoleucine, valine, phenylalanine, tryp- } \\
\text { tophan, plasma taurine and cystathionine } \\
\text { decreased, while glutamic acid and glutamine } \\
\text { increased after a FP }\end{array}$ \\
\hline
\end{tabular}




\begin{tabular}{|c|c|c|c|c|c|c|c|c|}
\hline$a / a$ & $\begin{array}{l}\text { Author(s), year, } \\
\text { country }\end{array}$ & Study design & Sample size $(n)$ & $\begin{array}{l}\text { Target age } \\
\text { groups } \\
\text { (years) }\end{array}$ & Fasting period & $\begin{array}{l}\text { Anthropometric mea- } \\
\text { surements }\end{array}$ & Arterial blood pressure & Haematologic data \\
\hline 10 & $\begin{array}{l}\text { Karras et al., } \\
\text { 2017, Greece }\end{array}$ & $\begin{array}{l}\text { Prospective } \\
\text { study }\end{array}$ & $50 \mathrm{~F}$ & $38 \cdot 8 \pm 9 \cdot 7$ & $\begin{array}{l}1 \text { fasting (Easter) } \\
\text { and } 1 \text { non-fast- } \\
\text { ing day }\end{array}$ & $\begin{array}{l}\mathrm{BMI} \text { and } \% \mathrm{BF} \text { were } \\
\text { within normal } \\
\text { range after a FP }\end{array}$ & NA & $\begin{array}{l}\text { F had normal total cholesterol, LDL and HDL- } \\
\text { cholesterol, triglycerides, glucose, insulin and } \\
\text { calcium levels and low vitamin D levels }\end{array}$ \\
\hline 11 & $\begin{array}{l}\text { Liali et al., } \\
2015, \text { Greece } \\
\text { (44) }\end{array}$ & $\begin{array}{l}\text { Prospective } \\
\text { study }\end{array}$ & $41 \mathrm{~F}$ (41 female) & $20-55$ & $48 \mathrm{~d}$, Easter FP & NA & NA & $\begin{array}{l}\text { F had increased PT and decreased APTT, } \\
\text { fibrinogen concentration and FVII activity }\end{array}$ \\
\hline 12 & $\begin{array}{l}\text { Papadaki et al., } \\
\text { 2011, Greece }\end{array}$ & $\begin{array}{l}\text { Prospective } \\
\text { study }\end{array}$ & $1 \mathrm{~F}$ & NA & $\begin{array}{l}1 \text { fasting (Easter) } \\
\text { and } 1 \text { non-fast- } \\
\text { ing week }\end{array}$ & NA & NA & NA \\
\hline 13 & $\begin{array}{l}\text { Chryssochoou } \\
\text { et al., 2010, } \\
\text { Greece }\end{array}$ & $\begin{array}{l}\text { Cross-sec- } \\
\quad \text { tional study }\end{array}$ & $\begin{array}{l}609 \text { children }(286 \\
\text { female, } 323 \\
\text { male) }\end{array}$ & $5-15 \cdot 5$ & NFP & $\begin{array}{l}12.1 \% \text { of children } \\
\text { who never fast } \\
\text { were obese in con- } \\
\text { trast to } 7.3 \% \text { of } \\
\text { those who fasted }\end{array}$ & NA & NA \\
\hline 14 & $\begin{array}{l}\text { Sarri et al., } \\
2009, \text { Greece }^{(38)}\end{array}$ & $\begin{array}{l}\text { Case-control } \\
\text { study }\end{array}$ & $\begin{array}{l}37 \mathrm{~F}(19 \text { female, } \\
18 \text { male) } \\
48 \mathrm{NF}(27 \text { female, } \\
21 \text { male })\end{array}$ & $\begin{array}{l}F 43 \cdot 0 \pm 13 \cdot 1 \\
N F \\
\quad 38 \cdot 6 \pm 9 \cdot 6\end{array}$ & $\begin{array}{l}40 \mathrm{~d} \text {, Christmas } \\
\text { FP }\end{array}$ & $\begin{array}{l}\mathrm{F} \text { had higher } \mathrm{BMI} \text { in } \\
\text { general }\end{array}$ & NA & $\begin{array}{l}\text { F decreased serum retinol, a-tocopherol, total } \\
\text { cholesterol, LDL-cholesterol and triacylgly- } \\
\text { cerol levels, and } \\
\alpha \text {-tocopherol:total cholesterol and retinol:total } \\
\text { cholesterol ratios }\end{array}$ \\
\hline 15 & $\begin{array}{l}\text { Papadaki et al., } \\
\text { 2007, Greece }\end{array}$ & $\begin{array}{l}\text { Prospective } \\
\text { study }\end{array}$ & $10 \mathrm{~F}$ & $37 \cdot 5 \pm 12 \cdot 3$ & $\begin{array}{l}1 \text { fasting (Easter) } \\
\text { and } 1 \text { non-fast- } \\
\text { ing week }\end{array}$ & $\begin{array}{l}\mathrm{F} \text { had increased BW } \\
\text { and BMI during a } \\
\text { NFP }\end{array}$ & $\begin{array}{l}\text { F had lower SBP and } \\
\text { DBP during a NFP }\end{array}$ & $\begin{array}{l}\text { F increased total cholesterol and LDL- and } \\
\text { HDL-cholesterol after a NFP, and had lower } \\
\text { total cholesterol:HDL ratio during a FP }\end{array}$ \\
\hline 16 & $\begin{array}{l}\text { Sarri et al., } \\
2007, \text { Greece }^{(37)}\end{array}$ & $\begin{array}{l}\text { Case-control } \\
\text { study }\end{array}$ & $\begin{array}{l}38 \mathrm{~F}(19 \text { female, } \\
19 \text { male) } \\
29 \mathrm{NF}(13 \text { female, } \\
16 \text { male })\end{array}$ & $\begin{array}{l}\mathrm{F} 41.3 \pm 11.9 \\
\mathrm{NF} \\
\quad 39.5 \pm 11 \cdot 3\end{array}$ & $\begin{array}{l}3 \text { FP; Christmas, } \\
\text { Lent and } \\
\text { Assumption }\end{array}$ & $\begin{array}{l}\text { F had higher BMI } \\
\text { and WC when } \\
\text { compared with } \\
\text { controls }\end{array}$ & $\begin{array}{l}\text { F had higher SBP and } \\
\text { DBP during all times. F } \\
\text { decreased their SBP } \\
\text { after all } 3 \text { FP }\end{array}$ & NA \\
\hline 17 & $\begin{array}{l}\text { Sarri et al., } \\
\text { 2005, Greece }\end{array}$ & $\begin{array}{l}\text { Case-control } \\
\text { study }\end{array}$ & $\begin{array}{l}35 \mathrm{~F}(18 \text { female, } \\
17 \text { male }) \\
24 \mathrm{NF}(13 \text { female, } \\
11 \text { male })\end{array}$ & $\begin{array}{l}\mathrm{F} 43 \cdot 6 \pm 13 \cdot 2 \\
\mathrm{NF} \\
\quad 39 \cdot 8 \pm 7 \cdot 6\end{array}$ & $\begin{array}{l}40 \mathrm{~d} \text {, Christmas } \\
\text { FP }\end{array}$ & NA & NA & $\begin{array}{l}\text { F had lower pre serum ferritin, higher pre } \\
\text { MCHC, higher pre TIBC and lower end TIBC. } \\
\text { Serum ferritin levels were increased after fasting } \\
\text { period in both groups }\end{array}$ \\
\hline 18 & $\begin{array}{l}\text { Sarri et al., } \\
2004 \text {, Greece }\end{array}$ & $\begin{array}{l}\text { Case-control } \\
\text { study }\end{array}$ & $\begin{array}{l}60 \mathrm{~F}(29 \text { female, } \\
31 \text { male) } \\
60 \mathrm{NF}(36 \text { female, } \\
24 \text { male) }\end{array}$ & $\begin{array}{l}\mathrm{F} 42 \pm 12 \\
\mathrm{NF} 38 \pm 9\end{array}$ & $\begin{array}{l}3 \text { FP; Christmas, } \\
\text { Lent and } \\
\text { Assumption }\end{array}$ & NA & NA & NA \\
\hline 19 & $\begin{array}{l}\text { Sarri et al., } \\
2003, \text { Greece }\end{array}$ & $\begin{array}{l}\text { Case-control } \\
\text { study }\end{array}$ & $\begin{array}{l}60 \mathrm{~F} \text { ( } 29 \text { female, } \\
31 \text { male) } \\
60 \mathrm{NF}(36 \text { female, } \\
24 \text { male) }\end{array}$ & $\begin{array}{l}\mathrm{F} 41 \pm 12 \\
\mathrm{NF} 38 \pm 9\end{array}$ & $\begin{array}{l}3 \text { FP; Christmas, } \\
\text { Lent and } \\
\text { Assumption }\end{array}$ & $\begin{array}{l}\text { Fasters dropped BMI } \\
\text { by } 1.4 \%\end{array}$ & NA & $\begin{array}{l}\text { F had } 12.5 \% \text { lower total cholesterol, } 15.9 \% \\
\text { lower LDL, and lower LDL/HDL ratio when } \\
\text { compared with controls. } \\
\text { Fasters had a } 9.1 \% \text { reduction in total choles- } \\
\text { terol, } 12.4 \% \text { reduction in LDL and } 8.5 \% \\
\text { reduction in HDL. } \\
\text { ApoE genotype distribution did not differ } \\
\text { between groups }\end{array}$ \\
\hline 20 & $\begin{array}{l}\text { Basilakis et al., } \\
\text { 2002, Greece }\end{array}$ & $\begin{array}{l}\text { Prospective } \\
\text { study }\end{array}$ & $\begin{array}{l}36 \mathrm{~F} \text { ( } 25 \text { female, } \\
11 \text { male) }\end{array}$ & $42 \cdot 4 \pm 14 \cdot 6$ & $\begin{array}{l}40 \mathrm{~d} \text {, Christmas } \\
\text { FP }\end{array}$ & $\begin{array}{l}\text { F dropped BMI and } \\
\text { BW by } 2-3 \% \text { and } \\
\text { triceps skin fold by } \\
10 \% \text { after a FP }\end{array}$ & NA & $\begin{array}{l}\text { F lowered total cholesterol, LDL- and HDL-cho- } \\
\text { lesterol, plasma urea and creatinine levels, } \\
\text { increased triacylglycerol levels and blood glu- } \\
\text { cose, iron and ferritin levels remained the } \\
\text { same after a FP }\end{array}$ \\
\hline
\end{tabular}

*NA, not applicable; F, faster; NF, non-faster; FP, fasting period; NFP, non-fasting period; BW, body weight; BMI, body mass index; BF, body fat; VF, visceral fat; PT, prothrombin time; APTT, activated partial thromboplastin time; FVII, coagulation factor VII; TAC, total antioxidant capacity. 
Table 3. Results of dietary intake

\begin{tabular}{|c|c|c|c|c|c|c|c|}
\hline$a / a$ & $\begin{array}{l}\text { Author(s), year, } \\
\text { country }\end{array}$ & $\begin{array}{l}\text { Dietary assessment } \\
\text { method }\end{array}$ & $\begin{array}{l}\text { Results in energy and food } \\
\text { intake }\end{array}$ & $\begin{array}{l}\text { Results in carbohydrate, protein and } \\
\text { fat intake }\end{array}$ & Results in rest macronutrients & $\begin{array}{l}\text { Results in miner- } \\
\text { als }\end{array}$ & Results in vitamins \\
\hline 1 & $\begin{array}{l}\text { Rodopaios et al., } \\
\text { 2020, Greece } \\
(33)\end{array}$ & $\begin{array}{l}\text { Three } 24-h \text { dietary } \\
\text { recall and food } \\
\text { frequency ques- } \\
\text { tionnaire }\end{array}$ & NA & $\mathrm{F}$ had lower protein and fat intake & NA & $\begin{array}{l}\text { F had lower cal- } \\
\text { cium, magne- } \\
\text { sium, } \\
\text { selenium, iron } \\
\text { and phospho- } \\
\text { rus intake }\end{array}$ & $\begin{array}{l}\mathrm{F} \text { had lower intake of } \\
\text { vitamin B3, B12, D, E } \\
\text { and folate }\end{array}$ \\
\hline 2 & $\begin{array}{l}\text { Rodopaios et al., } \\
\text { 2020, Greece } \\
(32)\end{array}$ & $\begin{array}{l}\text { Three 24-h dietary } \\
\text { recall and food } \\
\text { frequency ques- } \\
\text { tionnaire }\end{array}$ & $\begin{array}{l}\text { F consumed fewer servings } \\
\text { of dairy, soya products } \\
\text { and alcohol per week }\end{array}$ & $\mathrm{F}$ had lower protein intake & NA & $\begin{array}{l}\mathrm{F} \text { had lower cal- } \\
\text { cium intake }\end{array}$ & $\begin{array}{l}\text { F had lower vitamin } D \\
\text { intake }\end{array}$ \\
\hline 3 & $\begin{array}{l}\text { Bethancourt et al., } \\
\text { 2019, USA }\end{array}$ & $\begin{array}{l}\text { 7-d weighed food } \\
\text { records and food } \\
\text { frequency ques- } \\
\text { tionnaire }\end{array}$ & $\begin{array}{l}\text { F decreased red meat, } \\
\text { poultry, processed meat, } \\
\text { all dairy and egg con- } \\
\text { sumption and increased } \\
\text { fish, shellfish and plant- } \\
\text { based food intake after } \\
\text { FP }\end{array}$ & NA & NA & NA & NA \\
\hline 4 & $\begin{array}{l}\text { Bethancourt et al., } \\
\text { 2019b, USA }{ }^{(39)}\end{array}$ & $\begin{array}{l}\text { 7-d weighed food } \\
\text { records and food } \\
\text { frequency ques- } \\
\text { tionnaire }\end{array}$ & $\begin{array}{l}\text { F lowered their energy } \\
\text { intake, decreased meat, } \\
\text { dairy products and eggs } \\
\text { intake while increasing } \\
\text { legume, soya product, } \\
\text { nut and seed intake }\end{array}$ & $\begin{array}{l}F \text { reduced protein and fat and } \\
\text { increased carbohydrate intake }\end{array}$ & $\begin{array}{l}\text { F reduced animal protein and } \\
\text { increased plant protein intake, } \\
\text { reduced saturated fat, trans-fatty } \\
\text { acid and increased fibre intake }\end{array}$ & $\begin{array}{l}\text { F had lower } \\
\text { intake of cal- } \\
\text { cium, zinc and } \\
\text { higher intake } \\
\text { of magnesium }\end{array}$ & $\begin{array}{l}\text { F had lower intakes of } \\
\text { vitamin B12, D }\end{array}$ \\
\hline 5 & $\begin{array}{l}\text { Rodopaios et al., } \\
\text { 2019, Greece }\end{array}$ & $\begin{array}{l}\text { Three } 24-h \text { dietary } \\
\text { recall and food } \\
\text { frequency ques- } \\
\text { tionnaire }\end{array}$ & $\begin{array}{l}\text { F consumed less servings } \\
\text { of dairy and soya prod- } \\
\text { ucts per week. }\end{array}$ & $\mathrm{F}$ had lower protein intake & NA & $\begin{array}{l}\text { F had lower cal- } \\
\text { cium intake }\end{array}$ & $\begin{array}{l}\text { F had lower vitamin } D \\
\text { intake }\end{array}$ \\
\hline 6 & $\begin{array}{l}\text { Karras et al., 2019, } \\
\quad \text { Greece }{ }^{(46)}\end{array}$ & $\begin{array}{l}\text { 2-d weighed food } \\
\text { record }\end{array}$ & $\begin{array}{l}\text { Athonian monks had lower } \\
\text { energy intake during both } \\
\text { RD and NRD }\end{array}$ & $\begin{array}{l}\text { Athonian monks had lower fat and } \\
\text { carbohydrate intake, and higher } \\
\text { protein intake on a RD, while } \\
\text { higher carbohydrate, lower fat and } \\
\text { protein intake during a NRD }\end{array}$ & $\begin{array}{l}\text { Athonian monks had higher choles- } \\
\text { terol intake and lower saturated } \\
\text { fat intake on a RD. General F } \\
\text { had higher intake of saturated } \\
\text { fat and cholesterol intakes in a } \\
\text { NRD }\end{array}$ & $\begin{array}{l}\text { F had low intake } \\
\text { of calcium, } \\
\text { magnesium, } \\
\text { sodium and } \\
\text { potassium, } \\
\text { and adequate } \\
\text { iron intake }\end{array}$ & $\begin{array}{l}\text { Intake of vitamins } C \\
\text { and } B 12 \text { were higher } \\
\text { in Athonian monks, } \\
\text { while vitamins } D \text { and } \\
E \text { were below the } \\
\text { recommended in all } \\
F\end{array}$ \\
\hline 7 & $\begin{array}{l}\text { El-Sayed et al., } \\
\text { 2018, Egypt }\end{array}$ & NA & NA & NA & NA & NA & NA \\
\hline 8 & $\begin{array}{l}\text { Makedou et al., } \\
\text { 2018, Greece }\end{array}$ & NA & NA & NA & NA & NA & NA \\
\hline 9 & $\begin{array}{l}\text { Elshorbagy et al., } \\
\text { 2017, Egypt }{ }^{(47)}\end{array}$ & $\begin{array}{l}\text { Food frequency } \\
\text { questionnaire }\end{array}$ & NA & NA & NA & NA & NA \\
\hline 10 & $\begin{array}{l}\text { Karras et al., } 2017, \\
\quad \text { Greece }\end{array}$ & $\begin{array}{l}\text { 2-d weighed food } \\
\text { record }\end{array}$ & $\begin{array}{l}\text { F had lower total energy } \\
\text { intake in a RD }\end{array}$ & $\begin{array}{l}F \text { had higher fat and protein intake in } \\
\text { a RD }\end{array}$ & $\begin{array}{l}F \text { had high fibre intake during both } \\
\text { RD and NRD }\end{array}$ & $\begin{array}{l}\text { F had low intake } \\
\text { of calcium, } \\
\text { magnesium, } \\
\text { and sodium, } \\
\text { and adequate } \\
\text { iron intake }\end{array}$ & $\begin{array}{l}F \text { had low intake of vita- } \\
\text { mins } A, B 2 \text { and } D, \\
\text { while vitamins } B 12 \\
\text { and } C \text { were adequate } \\
\text { during both } R D \text { and } \\
\text { NRD }\end{array}$ \\
\hline 11 & $\begin{array}{l}\text { Liali et al., 2015, } \\
\text { Greece }{ }^{(44)}\end{array}$ & NA & NA & NA & NA & NA & NA \\
\hline 12 & $\begin{array}{l}\text { Papadaki et al., } \\
\text { 2011, Greece }\end{array}$ & $\begin{array}{r}\text { 7-d weighed food } \\
\text { record during } 1\end{array}$ & $\begin{array}{l}F \text { reduced total energy } \\
\text { intake during a FP }\end{array}$ & $\begin{array}{l}F \text { had increased fat, } \omega-3 \omega-6 \text { fatty } \\
\text { acids, and reduced saturated, }\end{array}$ & $\begin{array}{l}\mathrm{F} \text { had increased fibre and choles- } \\
\text { terol intake }\end{array}$ & $\begin{array}{l}F \text { had increased } \\
\text { iron and }\end{array}$ & $\begin{array}{l}F \text { had reduced vitamin } \\
E \text { intake }\end{array}$ \\
\hline
\end{tabular}


Table 3. (Continued)

Results in miner-

\begin{tabular}{|c|c|c|c|c|c|c|c|}
\hline $\mathrm{a} / \mathrm{a}$ & $\begin{array}{l}\text { Author(s), year, } \\
\text { country }\end{array}$ & $\begin{array}{l}\text { Dietary assessment } \\
\text { method }\end{array}$ & $\begin{array}{l}\text { Results in energy and food } \\
\text { intake }\end{array}$ & $\begin{array}{l}\text { Results in carbohydrate, protein and } \\
\text { fat intake }\end{array}$ & Results in rest macronutrients & $\begin{array}{l}\text { Results in miner- } \\
\text { als }\end{array}$ & Results in vitamins \\
\hline & & $\begin{array}{l}\text { fasting and } 1 \\
\text { non-fasting week }\end{array}$ & & $\begin{array}{l}\text { trans and mono-unsaturated fatty } \\
\text { acids intake }\end{array}$ & & $\begin{array}{l}\text { sodium intake, } \\
\text { while } \\
\text { decreased cal- } \\
\text { cium intake }\end{array}$ & \\
\hline 13 & $\begin{array}{l}\text { Chryssochoou } \\
\text { et al., 2010, } \\
\text { Greece }\end{array}$ & $\begin{array}{l}\text { 3-d weighed food } \\
\text { record }\end{array}$ & $\begin{array}{l}\text { Children who never fast had } \\
\text { higher energy intake }\end{array}$ & $\begin{array}{l}\text { Children who never fast had higher } \\
\text { saturated fatty acids, trans fatty } \\
\text { acids. Children who fast had } \\
\text { higher carbohydrate, lower fat, } \\
\text { saturated fatty acids and mono- } \\
\text { unsaturated fatty acids intake }\end{array}$ & $\begin{array}{l}\text { Children who never fast had higher } \\
\text { cholesterol intake }\end{array}$ & $\begin{array}{l}\text { Children who fast } \\
\text { had lower } \\
\text { intake of mag- } \\
\text { nesium }\end{array}$ & $\begin{array}{l}\text { Children who fast had } \\
\text { lower intake of vita- } \\
\text { min E }\end{array}$ \\
\hline 14 & $\begin{array}{l}\text { Sarri et al., 2009, } \\
\quad \text { Greece }{ }^{(38)}\end{array}$ & $\begin{array}{l}\text { One } 24-\mathrm{h} \text { recall and } \\
3-\mathrm{d} \text { weighed food } \\
\text { record, before } \\
\text { and after the } 3 \\
\text { FP }\end{array}$ & $\begin{array}{l}\text { F consumed more cereals, } \\
\text { rice or pasta after FP }\end{array}$ & $\begin{array}{l}F \text { increased carbohydrate and } \\
\text { decreased fat intake. }\end{array}$ & $\begin{array}{l}\mathrm{F} \text { increased their fibre and } \\
\text { decreased their cholesterol } \\
\text { intake after FP }\end{array}$ & $\begin{array}{l}\text { F consumed less } \\
\text { calcium, phos- } \\
\text { phorus and } \\
\text { sodium }\end{array}$ & $\begin{array}{l}F \text { consumed less vita- } \\
\text { mins B1, B6, niacin }\end{array}$ \\
\hline 15 & $\begin{array}{l}\text { Papadaki et al., } \\
\text { 2007, Greece }\end{array}$ & $\begin{array}{l}\text { 7-d weighed food } \\
\text { record during } 1 \\
\text { fasting and } 1 \\
\text { non-fasting week }\end{array}$ & $\begin{array}{l}\text { F consumed more legumes, } \\
\text { fish, shellfish, snails and } \\
\text { nuts during a FP }\end{array}$ & $\begin{array}{l}\text { F increased carbohydrate intake and } \\
\text { reduced protein, fat, saturated fat } \\
\text { and trans fatty acids intake during } \\
\text { a FP }\end{array}$ & $\begin{array}{l}F \text { increased their fibre intake during } \\
\text { a FP }\end{array}$ & $\begin{array}{l}F \text { increased their } \\
\text { folate and } \\
\text { decreased } \\
\text { their calcium } \\
\text { intake during a } \\
\text { FP }\end{array}$ & NA \\
\hline 16 & $\begin{array}{l}\text { Sarri et al., 2007, } \\
\quad \text { Greece }{ }^{(37)}\end{array}$ & $\begin{array}{l}\text { One } 24-\mathrm{h} \text { recall and } \\
3-\mathrm{d} \text { weighed food } \\
\text { record, before } \\
\text { and after the } 3 \\
\text { FP }\end{array}$ & $\begin{array}{l}\text { F consumed more fruits and } \\
\text { vegetables after all } 3 \mathrm{FP}\end{array}$ & NA & $\begin{array}{l}F \text { increased their fibre intake after } \\
\text { all } 3 \text { FP }\end{array}$ & $\begin{array}{l}\text { F reduced } \\
\text { sodium and } \\
\text { calcium, and } \\
\text { increased } \\
\text { magnesium } \\
\text { intake after all } \\
3 \text { FP }\end{array}$ & NA \\
\hline 17 & $\begin{array}{l}\text { Sarri et al., 2005, } \\
\quad \text { Greece }^{(36)}\end{array}$ & $\begin{array}{l}\text { One } 24-\mathrm{h} \text { recall and } \\
3 \text {-d weighed food } \\
\text { record, before } \\
\text { and after the } 3 \\
\text { FP }\end{array}$ & $\begin{array}{l}\text { F decreased their energy } \\
\text { intake after the FP. F } \\
\text { consumed more pulses } \\
\text { pre- and post-FP }\end{array}$ & NA & $\begin{array}{l}F \text { increased fibre intake after the } \\
\text { FP }\end{array}$ & $\begin{array}{l}\text { F increased } \\
\text { dietary iron } \\
\text { intake after the } \\
\text { FP }\end{array}$ & $\begin{array}{l}\text { F increased their vita- } \\
\text { min } C \text { intake after the } \\
\text { FP }\end{array}$ \\
\hline 18 & $\begin{array}{l}\text { Sarri et al., 2004, } \\
\quad \text { Greece } e^{(35)}\end{array}$ & $\begin{array}{l}\text { One } 24-\mathrm{h} \text { recall and } \\
3-\mathrm{d} \text { weighed food } \\
\text { record, before } \\
\text { and after the } 3 \\
\text { FP }\end{array}$ & $\begin{array}{l}\mathrm{F} \text { decreased energy intake } \\
\text { after all } 3 \text { fasting periods. } \\
\mathrm{F} \text { consumed more pota- } \\
\text { toes, fruits and vegeta- } \\
\text { bles after all } 3 \mathrm{FP}\end{array}$ & $\begin{array}{l}\mathrm{F} \text { increased carbohydrate consump- } \\
\text { tion, reduced protein and fat } \\
\text { intake after all } 3 \mathrm{FP}\end{array}$ & $\begin{array}{l}\text { F decreased saturated fatty, trans- } \\
\text { fatty acids, dietary cholesterol } \\
\text { intake, and increased fibre } \\
\text { intake after all 3 FP. F } \\
\text { decreased the n6:n3 ratio by } 9 \\
\%\end{array}$ & $\begin{array}{l}\text { F had higher } \\
\text { intake of folate } \\
\text { and iron and } \\
\text { lower intake of } \\
\text { calcium after } \\
\text { FP }\end{array}$ & $\begin{array}{l}\text { No difference in vita- } \\
\text { mins } A, C, E, B 1, B 6, \\
B 12 \text { and niacin }\end{array}$ \\
\hline 19 & $\begin{array}{l}\text { Sarri et al., 2003, } \\
\quad \text { Greece }{ }^{(6)}\end{array}$ & $\begin{array}{l}\text { One } 24-\mathrm{h} \text { recall and } \\
3-\mathrm{d} \text { weighed food } \\
\text { record, before } \\
\text { and after the } 3 \\
\text { FP }\end{array}$ & $\begin{array}{l}\text { F had } 10 \% \text { reduction, } \\
\text { whereas NF had a } 7 \% \\
\text { increase in energy intake }\end{array}$ & $\begin{array}{l}\mathrm{F} \text { had } 23 \% \text { increase in carbohy- } \\
\text { drates and } 17 \% \text { reduction in fat }\end{array}$ & $\begin{array}{l}\text { F had } 43.5 \% \text { increase in fibre con- } \\
\text { sumption, while NF had } 3.3 \% \\
\text { increase }\end{array}$ & NA & NA \\
\hline 20 & $\begin{array}{l}\text { Basilakis et al., } \\
\text { 2002, Greece }\end{array}$ & $\begin{array}{l}\text { 7-d weighed food, } \\
\text { before and dur- } \\
\text { ing the last week } \\
\text { of fasting }\end{array}$ & $\begin{array}{l}\mathrm{F} \text { had } 20 \% \text { reduction in } \\
\text { energy intake during FP }\end{array}$ & $\begin{array}{l}\text { F had } 17 \% \text { reduction in carbohy- } \\
\text { drate, } 17 \% \text { reduction in protein } \\
\text { and } 33 \% \text { reduction in fat intake } \\
\text { during FP }\end{array}$ & $\begin{array}{l}\text { F had } 77 \% \text { reduction in choles- } \\
\text { terol, } 39 \% \text { reduction in satu- } \\
\text { rated fatty acids, } 36 \% \text { reduction } \\
\text { in polyunsaturated fatty acids } \\
\text { and no reduction in monoun- } \\
\text { saturated fatty acids during FP }\end{array}$ & NA & NA \\
\hline
\end{tabular}

*NA, not applicable; F, faster; NF, non-faster; FP, fasting period; NFP, non-fasting period; RD, restrictive day; NRD, non-restrictive day. 
According to Sarri and colleagues, after the three main COC fasting periods, mean systolic blood pressure was lowered in thirty-eight fasters when comparing with twenty-nine non-fasters. Further analysis revealed that fasters with high levels of blood pressure, during all fasting periods, were people of older age and with higher BMI, resulting in high levels of blood pressure $^{(37)}$. Also, another study showed that ten members of monasteries in Greece had higher values of systolic blood pressure after an Easter fasting week, probably as a result of lower calcium intake and/or the use of salt during fasting periods to improve the taste of meals ${ }^{(43)}$. Elsayed and colleagues reported that, after an Easter fasting period, fasters with and without T2DM decreased systolic blood pressure and increased diastolic blood pressure, and fasters with hypertension lowered both systolic blood pressure and diastolic blood pressure after the fasting period $^{(48)}$. A cross-sectional study in Greece demonstrated that both systolic and diastolic blood pressure did not differ between 100 fasters and 100 non-fasters ${ }^{(32)}$.

\section{Haematological outcomes}

Total cholesterol and LDL-cholesterol decreased by $9 \cdot 1 \%$ and $12 \cdot 4$ $\%$, respectively, and the LDL:HDL ratio was significantly lower after all three major fasting periods in Greece in sixty fasters who were members of monasteries. This could be a result of the $43.5 \%$ increase in fibre consumption and $17 \%$ reduction in fat intake, as shown from the 24-h recalls and the 3-d weighed food records collected from fasters during all fasting periods. Notably, in the same study, when fasters returned to their usual dietary habits, the levels of total cholesterol and LDL-cholesterol were increased ${ }^{(6)}$. During an Easter fasting period, in ninety-nine fasters in the United States, it was reported that each serving decrease in meat, dairy product and egg consumption, foods not allowed in this period, was associated with an average decrease of $3.7 \%$ in total cholesterol levels and $3.6 \%$ decrease in LDL-cholesterol ${ }^{(40)}$. Also, Papadaki and colleagues showed that, after an Easter fasting week, there were lower levels of total cholesterol and LDL-cholesterol, and the total-cholesterol:HDLcholesterol ratio was lower, probably as a result of increased fruit, vegetable, legume, fish, shellfish, snail and nut consumption as it was reported in 7-d weighed food records during the fasting period $^{(43)}$. Furthermore, total cholesterol ${ }^{(38,41,46,47)}$ and LDL-cholesterol ${ }^{(38,41,46)}$ were both decreased after different COC fasting periods, while HDL was found to be increased during a restrictive period $^{(46)}$ and lower after the Christmas fasting period ${ }^{(41)}$.

Triacylglycerol levels were reduced in fasters with $\mathrm{T} 2 \mathrm{DM}^{(48)}$ and in laypeople following the Christmas fasting period ${ }^{(38)}$, while one publication showed that triglycerides were not affected in addition to no changes in glucose metabolism and C-reactive protein levels (CRP) ${ }^{(40)}$.

Elsayed and colleagues focused their study on people with and without T2DM. Fasters with T2DM had no changes in fasting blood glucose, while some fasters without T2DM increased their fasting blood glucose levels after the fasting period. Some fasters with T2DM dropped their HbA1c levels, and no changes were found in total cholesterol or LDL- and HDL-cholesterol levels ${ }^{(48)}$.

After the Easter fasting period, blood sample analysis showed reduced leucine, isoleucine, valine, phenylalanine, tryptophan, taurine, cystathionine, glutamic acid and glutamine concentrations in fasters ${ }^{(47)}$. In addition, plasma retinol and $\alpha$-tocopherol concentrations, as well as $\alpha$-tocopherol:total cholesterol and retinol:total cholesterol ratios were all reduced after an Easter fasting period ${ }^{(38)}$. According to Basilakis et al., plasma protein, urea and creatinine levels were decreased and blood glucose remained the same, while iron and ferritin levels did not change in fasters after the Christmas fasting period ${ }^{(41)}$. Higher serum ferritin levels were also noted after the Christmas fasting period in both fasters and non-fasters ${ }^{(36)}$. In the same study, total ironbinding capacity (TIBC) and mean corpuscular $\mathrm{Hb}$ concentration (MCHC) were decreased in fasters ${ }^{(36)}$.

In a study focused on fifty Athonian monks, results showed that they had better fasting insulin concentrations and HOMAIR values, parathyroid hormone levels were higher, serum calcium was in normal range and all monks were vitamin $\mathrm{D}$ deficient. This was probably a result not only of the dietary intake, which was low in vitamin $\mathrm{D}$, but also because of the mandatory clothing of a black cassock they have to wear during outdoor activities ${ }^{(45)}$.

Coagulation factor VII (FVII) activity was found to be lower after a fasting period ${ }^{(44,49)}$. Also, after Easter fasting, prothrombin time (PT) was increased, while activated partial thromboplastin time (APTT) and fibrinogen concentration were lower when comparing with pre-fast values ${ }^{(44)}$. Another publication reported that, after Easter fasting, there was a decrease in leucocytes, granulocytes, haemoglobin, erythrocytes, mean erythrocyte volume (MCV) and platelets, while total antioxidant capacity (TAC) levels were increased ${ }^{(49)}$.

Lastly, one study found that ApoE genotype distribution did not differ between fasters and controls after different fasting periods ${ }^{(6)}$.

\section{Anthropometric parameters}

Following the Christmas fasting regimen, fasters dropped their body weight and BMI by 2-3\%, while the triceps skinfold was reduced by $10 \%{ }^{(41)}$. Among a Greek population following the three major fasting periods, there was a $1.4 \%$ decline in fasters' $\mathrm{BMI}^{(37)}$. Body weight was found to be lower in fasters when compared with non-fasters after a fasting period ${ }^{(40,43,47)}$, with same outcomes in $\mathrm{BMI}^{(43,48)}$, waist circumference ${ }^{(48)}$ and lean body mass ${ }^{(47)}$. In the only study with children participants, obesity was reported in $12.1 \%$ of those who never fasted in contrast to $7 \cdot 3 \%$ of children fasters ${ }^{(34)}$.

Rodopaios and colleagues reported that overweight and obese participants were over $50 \%$ in both fasters and non-fasters, an outcome that needs further investigation regarding the quantity of foods eaten during fasting periods apart from the quality $^{(31)}$. Also, in the same study, it was shown that bone mineral density and bone mineral content at the lumbar spine, right and left hip, and right and left femoral neck did not differ between fasters and non-fasters when evaluated with dualenergy X-ray absorptiometry ${ }^{(31,32)}$, which revealed a $4 \%$ incidence of osteoporosis in fasters compared with $14 \%$ in non-fasters, and bone mineral density was high in overweight and obese men and postmenopausal women over the age of 50 years who followed the COC fasting regimen, without the use of dietary supplements ${ }^{(33)}$. 


\section{Discussion}

This scoping review aimed to present available evidence regarding the impact of COC fasting on human health in relation to MetS. As it was shown in some publications, fasting periods were characterised by less daily energy intake when comparing with non-fasting periods $^{(6,35,36,39,41,42,46)}$, with some reporting a $10 \%{ }^{(6)}$ or even a $20 \%{ }^{(41)}$ reduction in energy intake. Low fat intake $e^{(6,33,35,38,41-43)}$, low saturated and trans fatty acid intake ${ }^{(35,42,43)}$, and low monounsaturated fatty acid and high $\omega-3$ and $\omega-6$ fatty acids ${ }^{(42)}$ intakes were shown for fasters following COC fasting regimes in different fasting periods. Increased complex carbohydrate and fibre ${ }^{(6,32,33,35,36,40)}$ intakes were noted in fasters. In terms of protein consumption, results were mixed; increased protein intake was noted in Athonian monks, probably due to the high intake of shellfish, seafood and snails ${ }^{(45,46)}$, and in fasters living in the United States, where an increase in plant protein was noted during fasting periods due to high intake of legumes, nuts and seeds ${ }^{(39)}$, while lower protein intake was shown in other studies ${ }^{(31-33,36,43)}$.

Also, it was shown that people who fasted according to COC recommendations ${ }^{(35-37,42)}$ had increased consumption of fruits, legumes and vegetables that had been associated with a protective effect against CVD, diabetes ${ }^{(50)}$ and blood pressure ${ }^{(51)}$. In a study with 486 females living in Tehran, Esmaillzadeh and colleagues showed that those with the highest intake of fruits and vegetables had a $34 \%$ and $30 \%$ reduced chance of having MetS, respectively, when comparing with those who did not consume the above foods ${ }^{(52)}$. Also, as shown in the EPIC-InterAct prospective cohort study, with a representative sample of 16 154 participants and 11 years of follow-up, consumption of a great variety of root and green leafy vegetables was associated with lower risk of diabetes in Europe ${ }^{(53)}$. In the PREDIMED study, among 808 participants with high cardiovascular risk, those who had the highest adherence to the Mediterranean diet had $56 \%$ lower odds ratios of having MetS ${ }^{(26)}$. Furthermore, research from the Singapore Chinese Health Study, with 43176 participants and 11 years of follow-up, indicated that a dietary pattern with high intake of fruits, vegetables and soy products is linked with lower risk of diabetes, while a dietary pattern characterised by higher consumption of meat and processed meat has been linked with higher risk of diabetes in China ${ }^{(54)}$. As far as the current popular intermittent fasting is concerned, limited evidence exist about its effects in people with diabetes; most of its results are similar to those of caloric restrictions, and it should be followed only with the involvement of a physician, according to a recent review by Horne and colleagues ${ }^{(55)}$.

High fibre consumption has been observed to be associated with beneficial effects on waist circumference and HDL-cholesterol, both components of $\mathrm{MetS}^{(56)}$. Notably, in the ATTICA study, the Mediterranean diet was associated with $13 \%$ lower likelihood of having the MetS, as shown in 3042 participants in Greece ${ }^{(57)}$. Olive oil, which is allowed during some fasting periods, has been proven to have beneficial effects on lowering blood pressure, reducing plasma glucose and improving the total-cholesterol:HDL-cholesterol ratio, all different variables of $\mathrm{MetS}^{(58)}$. Nuts have been shown to reduce plasma cholesterol, triacylglycerol and waist circumference, all being variables of MetS ${ }^{(59)}$. Lastly, another study has shown that $84 \mathrm{~g}$ of nut consumption per day has beneficial effects on body fat levels and body weight reduction ${ }^{(60)}$.

In addition, findings from the COC fasting studies are similar to Adventist Health Study 2 (AHS-2) results, in which 38118 strict, lacto-ovo-, pesco- and semi-vegetarians and 33634 nonvegetarians were included. AHS-2 results revealed that vegetarians had reduced calorie intake, and had higher total fibre and plant protein intake as a consequence of increased fruit, vegetable, legume and nut consumption, and higher intake of $\omega$ - 3 fatty acids due to consumption of fishes, when compared with nonvegetarians. Results have shown that vegetarians have a significantly lower risk of having MetS when compared with non-vegetarians $^{(61,62)}$. Similarly, 9850 adults over 19 years old participated in the Korean National Health and Nutrition Examination Survey, where it was shown that a dietary pattern that included grains, vegetables and fish was associated with a $14 \%$ reduced risk of $\mathrm{MetS}^{(63)}$. One recent review and meta-analysis of different dietary patterns showed that adherence to a 'healthy' diet pattern, mainly characterised by high consumption of fruits, vegetables, whole grains, poultry, fish, nuts, legumes and low-fat dairy products, significantly decreased MetS risk by $15 \%$, when compared with a 'meat/western' diet pattern, which increased MetS risk by $19 \%$ and was characterised by high intake of red and processed meat, eggs, refined grains and sweets ${ }^{(64)}$.

The effects of COC fasting period on blood pressure results were mixed. Two publications reported no changes in both systolic blood pressure and diastolic blood pressure ${ }^{(31,32)}$, and one showed low levels of systolic blood pressure under all fasting periods $^{(37)}$. Also, Papadaki et al. showed that both systolic blood pressure and diastolic blood pressure were lower only in non-fasting periods ${ }^{(43)}$. Taking into consideration that salt intake was never investigated in any study, high blood pressure could be possibly be due to the addition of salt during cooking in favour of taste ${ }^{(43)}$. Also, it could be a result of low calcium intake during COC fasting periods, given that calcium has been associated with blood pressure control ${ }^{(65)}$. In some cases, this could be due to the undiagnosed prevalence of arterial hypertension as people under religious orders living in monasteries might have limited access to health care services ${ }^{(37,66)}$. Results from the SUN cohort study, in 9408 participants with a follow-up of 6 years, showed that people with higher adherence to the Mediterranean diet had lower mean levels of systolic and diastolic blood pressure when compared with those with the lowest adherence in Spain ${ }^{(67)}$. Similarly, in Greece, the results of the EPIC study indicated that adherence to the Mediterranean diet is inversely associated with blood pressure levels ${ }^{(68)}$, and the ATTICA study showed that a food pattern characterised by consumption of fish, vegetables, fruits, legumes and cereals was inversely associated with blood pressure $^{(57)}$. What is more, among 6627 vegans, vegetarians and fish eaters and 4737 meat eaters participating in the EPICOxford study in the UK, vegans had the lowest prevalence of hypertension and the lowest mean systolic and diastolic blood pressure, while meat eaters had the highest values ${ }^{(69)}$. Same findings were shown in 500 non-Black individuals in AHS- $2^{(70)}$, as well as in 592 Black individuals in AHS-2 across the United States and Canada ${ }^{(71)}$, where vegans had significantly lower values in systolic and diastolic blood pressure when compared with non-vegetarians. In contrast, blood pressure was not different for people 
following alternate-day fasting, which accounts for one fast day and one usual intake, for 6 and 12 months when comparing with a control group ${ }^{(72)}$. Also, a similar reduction in both systolic and diastolic blood pressure was observed in premenopausal women following intermittent fasting and in those who followed a lowcalorie $\operatorname{diet}^{(73)}$

A potential benefit for lipid profile is indicated, as total cholesterol and LDL-cholesterol concentrations were reduced at the end of COC fasting periods ${ }^{(6,38,40,41,43,45,47)}$. Some studies showed a reduction in HDL-cholesterol concentrations ${ }^{(6,41,46)}$, a finding that is common in low-fat and vegetarian diets ${ }^{(74,75)}$. Similar results were shown in the PREDIMED study, where participants with higher adherence to the Mediterranean diet had 54\% lower odds of having high triacylglycerol levels ${ }^{(26)}$ and $47 \%$ lower odds of having low HDL-cholesterol levels ${ }^{(27)}$, both MetS criteria. The EPIC-Oxford study, with 11004 participants in the UK, showed that mean LDL-cholesterol was $12 \%$ lower in vegetarians than in omnivores $^{(69)}$. Also, the ATTICA study in Greece, with 3042 participants, showed that the Mediterranean diet was inversely associated with triacylglycerol levels and positively associated with HDL-cholesterol levels ${ }^{(57)}$

Fasting blood glucose has not been investigated thoroughly, apart from one study with participants having both lower and higher fasting blood glucose levels after a fasting period ${ }^{(48)}$. The Tzu Chi Health Study, with 4384 Taiwanese participants, found that vegetarian diet characterised by increased intake of soy products, vegetables, nuts and whole-grain products was negatively associated with impaired fasting blood glucose. The prevalence of diabetes was $0.6 \%$ in vegetarian pre-menopausal women versus $2.3 \%$ in meat eater ones, $2.8 \%$ versus $10 \%$ in vegetarian menopausal women versus meat eaters, and $4.3 \%$ and $8.1 \%$ in vegetarian men and omnivores, respectively $^{(76)}$. Lower prevalence and incidence of diabetes were also found in 41387 vegetarians from the United States and Canada who participated in the AHS-2 study ${ }^{(77)}$.

Results concerning body weight and BMI are limited. Studies showed a reduction in body weight ${ }^{(40,41,43)}$ and $\mathrm{BMI}^{(43,47,48)}$, while one showed optimal body weight in fasters before and after all fasting periods ${ }^{(45)}$, but others do not report any data, meaning that conclusions cannot be drawn. Similarly, due to the effect of low energy intake in vegetarian diets, people who follow these diets have lower BMI when compared with meat eaters ${ }^{(78)}$. Furthermore, Mediterranean diet seems to contribute to weight loss and maintaining a healthy body weight ${ }^{(79,80)}$. Also, results from the EPIC-Oxford ${ }^{(81)}$, AHS-2 study $^{(61)}$ and Tzu Chi Health Study ${ }^{(76)}$ showed that vegetarians had lower BMI when compared with non-vegetarian people, and these differences were reflected in a lower prevalence of obesity in vegetarians. In general, it has been observed that vegetarians are leaner than omnivores ${ }^{(82,83)}$, probably due to high intake of fibre in these diets ${ }^{(81,84)}$. Trepanowski and colleagues showed in a recent randomised clinical trial that participants who followed the strategy of alternate-day fasting did not have a significantly different weight loss after 6 and 12 months when compared with people who did not fast ${ }^{(72)}$.

Although studies regarding COC fasting regimen were carried out in three different countries, that is, Greece, United States and Egypt, the main results were similar and demonstrated low energy intake, low fat intake, low animal protein and high vegetable protein intake, high complex carbohydrate and fibre intake, and low calcium and vitamin D intake in laypeople following the COC fasting guidelines during different fasting periods. It is of great importance to mention that the fifth-largest Orthodox population in the world is found in Greece, followed by Egypt in tenth place and the United States in sixteenth ${ }^{(85)}$. It should also be mentioned that different variants of the COC fasting diet are included in the review, from Cretan and Mediterranean diet where meat consumption is allowed in non-fasting periods, to Athonian diet where meat is not allowed at all, but fish, seafood and snails are allowed. This contributes to the increased protein intake in Athonian monks seen throughout the year ${ }^{(46)}$.

Results from available studies with participants following the COC fasting recommendations revealed some positive outcomes on different variables of the MetS, although conclusions cannot be drawn on individual studies regarding any aspect of health.

\section{Limitations}

Our scoping review has several limitations which have to be taken into consideration when results are being evaluated, with some already being mentioned. First, the number of available and included articles is low, with small number of subjects involved from the same research group ${ }^{(6,31,32,35-40,42,43,45,46)}$. There is lack of control group in eleven out of twenty publications investigating the health effects of COC fasting regimen ${ }^{(34,39-47,49)}$ and the possibility of under- or over-estimating dietary intake either when subjects report it on questionnaires or when investigators analyse the information on available nutrition software in sixteen studies $^{(6,31,32,34-43,45-47)}$, which are among the confounding variables that influence the cause-and-effect relationship we are noticing. Moreover, the time intervals during which fasters were followed were short with no long-term follow-up period in all included articles. Also, the level of adherence and the years of compliance with the COC fasting guidelines were different in most of the included articles. No study was focused on foods eaten during non-fasting periods that might affect the results of studies investigating the long-term results of COC fasting regimen, and heterogeneity was high regarding design method and study sample. Finally, the included studies focus on different risk factors of MetS, meaning that results are interpreted individually and we cannot draw a clear conclusion.

\section{Conclusion}

The COC fasting diet pattern could be advised for the prevention of chronic diseases, as well as for people who want to follow a plant-based diet in terms of a healthier and/or more sustainable way of living. As already mentioned, the COC fasting dietary pattern has been the origin and the main characteristic of the diet of Crete since the 1960s, and was later called the Mediterranean diet. Adherence to the traditional diet of Crete is the main way of decreasing the MetS score. Thus, the COC fasting dietary pattern or its most beneficial components together with physical activity may be effective advice to decrease the MetS score. 
Although evidence in favour of health benefits is available, the COC fasting dietary recommendations should always be followed under personalised guidance on proper meal planning, like any other dietary pattern. Adding to this, the quality and the quantity of foods consumed during fasting and non-fasting periods have to be examined thoroughly. What is more, longterm follow-up studies should be carried out to investigate if the positive effects on health parameters are sustainable over time.

Further investigation is needed in relation to several health parameters, disease indices that are influenced by diet and in diverse ethnic populations, in order to assess the impact of this type of periodic vegetarianism that includes fish and seafood on nutritional status and chronic diseases. Taking into consideration the significant impact it has on public health, future studies should be focused on the interaction between COC fasting regimen and all risk factors of MetS.

\section{Financial support}

This research received no specific grant from any funding agency, commercial or not-for-profit sectors.

\section{Author contributions}

Anna Kokkinopoulou: Conceptualisation, Methodology, Data collection, Data analysis, Data interpretation, Writing original draft, Writing - review \& editing, Visualisation, Final approval of the submitted version

Anthony Kafatos: Conceptualisation, Methodology, Data collection, Data analysis, Data interpretation, Writing original draft, Writing - review \& editing, Visualisation, Final approval of the submitted version, Supervision

There are no conflicts of interest.

\section{Supplementary material}

To view supplementary material for this article, please visit https://doi.org/10.1017/S0954422421000184

\section{References}

1. Trepanowski JF \& Bloomer RJ. (2010) The impact of religious fasting on human health. NutrJ $9,57$.

2. Trepanowski JF, Canale RE, Marshall KE, Kabir MM \& Bloomer RJ. (2011) Impact of caloric and dietary restriction regimens on markers of health and longevity in humans and animals: a summary of available findings. Nutr J 10, 107.

3. Lazarou C \& Matalas A-L. (2010) A critical review of current evidence, perspectives and research implications of diet-related traditions of the Eastern Christian Orthodox Church on dietary intakes and health consequences. Int J Food Sci Nutr 61, 739-758.

4. Persynaki A, Karras S \& Pichard C. (2017) Unraveling the metabolic health benefits of fasting related to religious beliefs: a narrative review. Nutrition 35, 14-20.

5. Rodopaios NE, Manolarakis G, Peppas C \& Kafatos A. (2017) Is periodic abstinence from dairy products for half a year, detrimental to bone health of children and adolescents? Austin J Nutr Food Sci 5, 1094.

6. Sarri KO, Tzanakis NE, Linardakis MK, Mamalakis GD \& Kafatos AG. (2003) Effects of Greek Orthodox Christian Church fasting on serum lipids and obesity. BMC Public Health 3, 16.

7. Koufakis T, Karras SN, Antonopoulou V, Angeloudi E, Zebekakis P \& Kotsa K. (2017) Effects of Orthodox religious fasting on human health: a systematic review. Eur J Nutr $\mathbf{5 6}$, 2439-2455.

8. Trichopoulou A, Kouris-Blazos A, Wahlqvist ML, et al. (1995) Diet and overall survival in elderly people. BMJ 311, 14571460.

9. Keys A, Menotti A, Karvonen MJ, et al. (1986) The diet and 15year death rate in the seven countries study. Am J Epidemiol 124: 903-915.

10. Hatzis CM, Sifaki-Pistolla D \& Kafatos AG. (2015) History of the Cretan cohort of the Seven Countries Study. Horm Athens Greece 14, 326-329.

11. Aravanis C, Corcondilas A, Dontas AS, Lekos D \& Keys A. (1970) Coronary heart disease in seven countries. IX. The Greek islands of Crete and Corfu. Circulation $\mathbf{4 1}$ (4 Suppl), I88-I100.

12. Sarri K \& Kafatos A. (2005) The seven countries study in Crete: olive oil, Mediterranean diet or fasting? Public Health Nutr $\mathbf{8}$, 666.

13. Cannon G. (2004) Out of the Christmas box. Public Health Nutr 7, 987-990.

14. Hatzis CM, Papandreou C, Patelarou E, et al. (2013) A 50-year follow-up of the Seven Countries Study: prevalence of cardiovascular risk factors, food and nutrient intakes among Cretans. Horm Athens Greece 12, 379-385.

15. Trichopoulou A, Bamia C \& Trichopoulos D. (2009) Anatomy of health effects of Mediterranean diet: Greek EPIC prospective cohort study. BMJ 338, b2337.

16. Saklayen MG. (2018) The Global epidemic of the metabolic syndrome. Curr Hypertens Rep 20, 12.

17. Alberti KG \& Zimmet PZ. (1998) Definition, diagnosis and classification of diabetes mellitus and its complications. Part 1: diagnosis and classification of diabetes mellitus provisional report of a WHO consultation. Diabet Med J Br Diabet Assoc 15, 539-553.

18. National Cholesterol Education Program (NCEP). (2002) Expert panel on detection, evaluation, and treatment of high blood cholesterol in adults (Adult Treatment Panel III). Third report of the National Cholesterol Education Program (NCEP) expert panel on detection, evaluation, and treatment of high blood cholesterol in adults (Adult Treatment Panel III) final report. Circulation 106, 3143-3421.

19. Alberti KGMM, Zimmet P \& Shaw J, IDF Epidemiology Task Force Consensus Group. (2005) The metabolic syndrome - a new worldwide definition. Lancet Lond Engl 366, 1059-1062.

20. Alberti KGMM, Eckel RH, Grundy SM, et al. (2009) Harmonizing the metabolic syndrome: a joint interim statement of the International Diabetes Federation Task Force on Epidemiology and Prevention; National Heart, Lung, and Blood Institute; American Heart Association; World Heart Federation; International Atherosclerosis Society; and International Association for the Study of Obesity. Circulation 120, 1640-1645.

21. Athyros VG, Mikhailidis DP, Papageorgiou AA, et al. (2004) Prevalence of atherosclerotic vascular disease among subjects with the metabolic syndrome with or without diabetes mellitus: the METS-GREECE Multicentre Study. Curr Med Res Opin 20, 1691-1701.

22. Panagiotakos DB, Pitsavos C, Chrysohoou C, et al. (2004) Impact of lifestyle habits on the prevalence of the metabolic 
syndrome among Greek adults from the ATTICA study. Am Heart J 147, 106-112.

23. Expert Panel on Detection, Evaluation, and Treatment of High Blood Cholesterol in Adults. (2001) Executive summary of The Third Report of The National Cholesterol Education Program (NCEP) Expert panel on detection, evaluation, and treatment of high blood cholesterol in adults (Adult Treatment Panel III). JAMA 285, 2486-2497.

24. Stensvold D, Viken H, Steinshamn SL, et al. (2020) Effect of exercise training for five years on all cause mortality in older adults - the Generation 100 study: randomised controlled trial. BMJ 371, m3485.

25. Tortosa A, Bes-Rastrollo M, Sanchez-Villegas A, BasterraGortari FJ, Nuñez-Cordoba JM \& Martinez-Gonzalez MA. (2007) Mediterranean diet inversely associated with the incidence of metabolic syndrome: the SUN prospective cohort. Diabetes Care 30, 2957-2959.

26. Babio N, Bulló M, Basora J, et al. (2009) Adherence to the Mediterranean diet and risk of metabolic syndrome and its components. Nutr Metab Cardiovasc Dis 19, 563-570.

27. Babio N, Toledo E, Estruch R, et al. (2014) Mediterranean diets and metabolic syndrome status in the PREDIMED randomized trial. CMAJ 186, E649-E657.

28. Munn Z, Peters MDJ, Stern C, Tufanaru C, McArthur A \& Aromataris E. (2018) Systematic review or scoping review? Guidance for authors when choosing between a systematic or scoping review approach. BMC Med Res Methodol 18, 143.

29. Moher D, Liberati A, Tetzlaff J, Altman DG, PRISMA Group. (2009) Preferred reporting items for systematic reviews and meta-analyses: the PRISMA statement. PLoS Med 6, e1000097.

30. Hawker S, Payne S, Kerr C, Hardey M \& Powell J. (2002) Appraising the evidence: reviewing disparate data systematically. Qual Health Res 12, 1284-1299.

31. Rodopaios NE, Mougios V, Konstantinidou A, et al. (2019) Effect of periodic abstinence from dairy products for approximately half of the year on bone health in adults following the Christian Orthodox Church fasting rules for decades. Arch Osteoporos 14, 68.

32. Rodopaios NE, Mougios V, Koulouri A-A, et al. (2020) Bone status of young adults with periodic avoidance of dairy products since childhood. Eur J Pediatr 179, 645-651.

33. Rodopaios NE, Manolarakis GE, Koulouri A-A, et al. (2020) The significant effect on musculoskeletal metabolism and bone density of the Eastern Mediterranean Christian Orthodox Church fasting. Eur J Clin Nutr 74, 1-7.

34. Chryssochoou E, Linardakis M, Chatziagorou E, Tsanakas I \& Kafatos A. (2010) The effect of Christian Orthodox Church fasting on the health and growth of children and adolescents. Pediatrics 73, 121-129.

35. Sarri KO, Linardakis MK, Bervanaki FN, Tzanakis NE \& Kafatos AG. (2004) Greek Orthodox fasting rituals: a hidden characteristic of the Mediterranean diet of Crete. Br J Nutr 92, 277-284.

36. Sarri KO, Kafatos AG \& Higgins S. (2005) Is religious fasting related to iron status in Greek Orthodox Christians? Br J Nutr 94, 198-203.

37. Sarri K, Linardakis M, Codrington C, Kafatos A. (2007) Does the periodic vegetarianism of Greek Orthodox Christians benefit blood pressure? Prev Med 44, 341-348.

38. Sarri K, Bertsias G, Linardakis M, Tsibinos G, Tzanakis N \& Kafatos A. (2009) The effect of periodic vegetarianism on serum retinol and alpha-tocopherol levels. Int J Vitam Nutr Res 79, 271-280.

39. Bethancourt HJ, Kratz M \& O'Connor K. (2019) Spiritually motivated restrictions on animal products have a limited impact on consumption of healthy plant-based foods. Br J Nutr 122, 808-819.
40. Bethancourt HJ, Kratz M \& O'Connor K. (2019)A short-term religious "fast" from animal products has a minimal impact on cardiometabolic health biomarkers irrespective of concurrent shifts in distinct plant-based food groups. Am J Clin Nutr 110, 722-732.

41. Basilakis A, Kiprouli K, Mantzouranis S, et al. (2002) Nutritional study in Greek-Orthodox Monasteries - effect of a 40-day religious fasting. Aktuel Ernäbrungsmed 27, 250-255.

42. Papadaki A, Valsta LM, Lampi AM, et al. (2011) Differences in nutrient intake during a Greek Orthodox Christian fasting and non-fasting week, as assessed by a food composition database and chemical analyses of 7-day weighed food samples. J Food Compos Anal 24, 22-28.

43. Papadaki A, Vardavas C, Hatzis C \& Kafatos A. (2007) Calcium, nutrient and food intake of Greek Orthodox Christian monks during a fasting and non-fasting week. Public Health Nutr 11, 1022-1029.

44. Liali M, Mpirintzis L, Vagdatli E, et al. (2015) The effects of Orthodox Christian Fasting on blood coagulation. Int $J$ Biomed Lab Sci 4, 39-43.

45. Karras SN, Persynaki A, Petróczi A, et al. (2017) Health benefits and consequences of the Eastern Orthodox fasting in monks of Mount Athos: a cross-sectional study. Eur J Clin Nutr 71, 743-749.

46. Karras SN, Koufakis T, Petróczi A, et al. Christian Orthodox fasting in practice: a comparative evaluation between Greek Orthodox general population fasters and Athonian monks. Nutrition 2019; 59, 69-76.

47. Elshorbagy A, Jernerén F, Basta M, et al. (2017) Amino acid changes during transition to a vegan diet supplemented with fish in healthy humans. Eur J Nutr 56, 1953-1962.

48. Elsayed A, Noreldin AKA, Elsamman MK, Zaky DS \& Kaldas ES. (2018) Impact of Christians fasting in type 2 diabetic patients among Egyptian Coptic Orthodox. J Diabetol 9, 88-94.

49. Makedou KG, Vagdatli E, Patziarela E, Konstantinidou V, Poimenidou E \& Lymperaki E. (2018) Total antioxidant capacity, haematological and coagulation parameters after Orthodox Christian Fast. Open Access Maced J Med Sci 6, 284-286.

50. Hermsdorff HHM, Zulet MA, Puchau B \& Martínez JA. (2010) Fruit and vegetable consumption and proinflammatory gene expression from peripheral blood mononuclear cells in young adults: a translational study. Nutr Metab 7, 42.

51. Perez-Vizcaino F, Duarte J, Jimenez R, Santos-Buelga C \& Osuna A. (2009) Antihypertensive effects of the flavonoid quercetin. Pharmacol Rep 61, 67-75.

52. Esmaillzadeh A, Kimiagar M, Mehrabi Y, Azadbakht L, Hu FB \& Willett WC. (2006) Fruit and vegetable intakes, C-reactive protein, and the metabolic syndrome. Am J Clin Nutr 84, 1489-1497.

53. Cooper AJ, Forouhi NG, Ye Z, et al. (2012) Fruit and vegetable intake and type 2 diabetes: EPIC-InterAct prospective study and meta-analysis. Eur J Clin Nutr 66, 1082-1092.

54. Odegaard AO, Koh W-P, Butler LM, et al. (2011) Dietary patterns and incident type 2 diabetes in Chinese men and women: the Singapore Chinese Health Study. Diabetes Care 34 , 880-885.

55. Horne BD, Grajower MM \& Anderson JL. (2020) Limited evidence for the health effects and safety of intermittent fasting among patients with type 2 diabetes. JAMA 324, 341-342.

56. Sahyoun NR, Jacques PF, Zhang XL, Juan W \& McKeown NM. (2006) Whole-grain intake is inversely associated with the metabolic syndrome and mortality in older adults. Am J Clin Nutr 83, 124-131.

57. Panagiotakos DB, Pitsavos C, Skoumas Y \& Stefanadis C. (2007) The association between food patterns and the metabolic syndrome using principal components analysis: the ATTICA Study. J Am Diet Assoc 107, 979-987. 
58. López-Miranda J, Pérez-Jiménez F, Ros E, et al. (2010) Olive oil and health: summary of the II international conference on olive oil and health consensus report, Jaén and Córdoba (Spain) 2008. Nutr Metab Cardiovasc Dis 20, 284-294.

59. Alvarez León EE, Henríquez P \& Serra-Majem L. (2006) Mediterranean diet and metabolic syndrome: a cross-sectional study in the Canary Islands. Public Health Nutr 9, 1089-1098.

60. Wien MA, Sabaté JM, Iklé DN, Cole SE \& Kandeel FR. (2003) Almonds vs complex carbohydrates in a weight reduction program. Int J Obes Relat Metab Disord 27, 1365-1372.

61. Rizzo NS, Jaceldo-Siegl K, Sabate J \& Fraser GE. (2013) Nutrient profiles of vegetarian and nonvegetarian dietary patterns. $J$ Acad Nutr Diet 113, 1610-1619.

62. Rizzo NS, Sabaté J, Jaceldo-Siegl K \& Fraser GE. (2011) Vegetarian dietary patterns are associated with a lower risk of metabolic syndrome: the Adventist Health Study 2. Diabetes Care 34, 1225-1227.

63. Kim J \& Jo I. (2011) Grains, vegetables, and fish dietary pattern is inversely associated with the risk of metabolic syndrome in South Korean adults. J Am Diet Assoc 111, 1141-1149.

64. Fabiani R, Naldini G \& Chiavarini M. (2019) Dietary patterns and metabolic syndrome in adult subjects: a systematic review and meta-analysis. Nutrients 11, 2056.

65. Miller GD, Jarvis JK \& McBean LD. (2001) The importance of meeting calcium needs with foods. J Am Coll Nutr 20 (2 Suppl), 168S-185S.

66. Tedesco MA, Di Salvo G, Caputo S, et al. (2001) Educational level and hypertension: how socioeconomic differences condition health care. J Hum Hypertens 15, 727-731.

67. Núñez-Córdoba JM, Valencia-Serrano F, Toledo E, Alonso A \& Martínez-González MA. (2009) The Mediterranean diet and incidence of hypertension: the Seguimiento Universidad de Navarra (SUN) Study. Am J Epidemiol 169, 339-346.

68. Psaltopoulou T, Naska A, Orfanos P, Trichopoulos D, Mountokalakis T \& Trichopoulou A. (2004) Olive oil, the Mediterranean diet, and arterial blood pressure: the Greek European Prospective Investigation into Cancer and Nutrition (EPIC) study. Am J Clin Nutr 80, 1012-1018.

69. Appleby PN, Davey GK \& Key TJ. (2002) Hypertension and blood pressure among meat eaters, fish eaters, vegetarians and vegans in EPIC-Oxford. Public Health Nutr 5, 645-654.

70. Pettersen BJ, Anousheh R, Fan J, Jaceldo-Siegl K \& Fraser GE. (2012) Vegetarian diets and blood pressure among white subjects: results from the Adventist Health Study-2 (AHS-2). Public Health Nutr 15, 1909-1916.

71. Fraser G, Katuli S, Anousheh R, Knutsen S, Herring P \& Fan J. (2015) Vegetarian diets and cardiovascular risk factors in black members of the Adventist Health Study-2. Public Health Nutr 18, 537-545.

72. Trepanowski J, Kroeger C, Barnosky A, et al. (2017) Effect of alternate-day fasting on weight loss, weight maintenance, and cardioprotection among metabolically healthy obese adults: a randomized clinical trial [Internet]. Vol. 177, JAMA internal medicine. JAMA Intern Med; [cited 2020 Oct 14]. Available from: https://pubmed.ncbi.nlm.nih.gov/ 28459931/.

73. Harvie MN, Pegington M, Mattson MP, et al. (2011) The effects of intermittent or continuous energy restriction on weight loss and metabolic disease risk markers: a randomized trial in young overweight women. Int J Obes 35, 714-727.

74. Asztalos B, Lefevre M, Wong L, et al. (2000) Differential response to low-fat diet between low and normal HDL-cholesterol subjects. J Lipid Res 41, 321-328.

75. Wang F, Zheng J, Yang B, Jiang J, Fu Y \& Li D. (2015) Effects of vegetarian diets on blood lipids: a systematic review and metaanalysis of randomized controlled trials. J Am Heart Assoc 4, e002408.

76. Chiu THT, Huang H-Y, Chiu Y-F, et al. (2014) Taiwanese vegetarians and omnivores: dietary composition, prevalence of diabetes and IFG. PloS One $\mathbf{9}$, e88547.

77. Tonstad S, Stewart K, Oda K, Batech M, Herring RP \& Fraser GE. (2013) Vegetarian diets and incidence of diabetes in the Adventist Health Study-2. Nutr Metab Cardiovasc Dis 23, 292-299.

78. Key TJ, Appleby PN \& Rosell MS. (2006) Health effects of vegetarian and vegan diets. Proc Nutr Soc 65, 35-41.

79. Hassapidou M, Tziomalos K, Lazaridou S, et al. (2020) The Nutrition Health Alliance (NutriHeAl) study: a randomized, controlled, nutritional intervention based on Mediterranean diet in Greek municipalities. J Am Coll Nutr 39, 338-344.

80. Poulimeneas D, Anastasiou CA, Santos I, Hill JO, Panagiotakos DB \& Yannakoulia M. (2020) Exploring the relationship between the Mediterranean diet and weight loss maintenance: the MedWeight study. Br J Nutr 124, 1-19.

81. Davey GK, Spencer EA, Appleby PN, Allen NE, Knox KH \& Key TJ. (2003) EPIC-Oxford: lifestyle characteristics and nutrient intakes in a cohort of 33883 meat-eaters and 31546 non meat-eaters in the UK. Public Health Nutr 6, 259-269.

82. Spencer EA, Appleby PN, Davey GK \& Key TJ. (2003) Diet and body mass index in 38000 EPIC-Oxford meat-eaters, fish-eaters, vegetarians and vegans. Int J Obes Relat Metab Disord 27, 728-734.

83. Newby PK, Tucker KL \& Wolk A. (2005) Risk of overweight and obesity among semivegetarian, lactovegetarian, and vegan women. Am J Clin Nutr 81, 1267-1274.

84. Cade JE, Burley VJ, Greenwood DC, UK Women's Cohort Study Steering Group. (2004) The UK Women's Cohort study: comparison of vegetarians, fish-eaters and meat-eaters. Public Health Nutr 7, 871-878.

85. Pew Research Center. (2017) Orthodox Christianity in the 21st Century [Internet]. Available from: https://www.pewforum. org/2017/11/08/orthodox-christianity-in-the-21st-century/. 
Appendix 1: Results of the quality assessment tool for the qualitative studies $(n=20)$

\begin{tabular}{|c|c|c|c|c|c|c|c|c|c|c|c|}
\hline Study & $\begin{array}{l}\text { Abstract } \\
\text { and title }\end{array}$ & $\begin{array}{l}\text { Introduction } \\
\text { and aims }\end{array}$ & $\begin{array}{l}\text { Method } \\
\text { and data }\end{array}$ & Sampling & $\begin{array}{l}\text { Data } \\
\text { analysis }\end{array}$ & $\begin{array}{l}\text { Ethics } \\
\text { and bias }\end{array}$ & $\begin{array}{l}\text { Finding/ } \\
\text { results }\end{array}$ & $\begin{array}{l}\text { Transferability/ } \\
\text { generalisability }\end{array}$ & $\begin{array}{l}\text { Implications } \\
\text { and usefulness }\end{array}$ & Total & Grade \\
\hline $\begin{array}{l}\text { Rodopaios et al., } \\
2020^{(33)}\end{array}$ & 4 & 4 & 4 & 4 & 4 & 3 & 4 & 4 & 3 & 34 & A \\
\hline $\begin{array}{l}\text { Rodopaios et al., } \\
2020^{(32)}\end{array}$ & 4 & 4 & 4 & 4 & 4 & 3 & 4 & 4 & 3 & 34 & A \\
\hline $\begin{array}{l}\text { Bethancourt et al., } \\
2019^{(40)}\end{array}$ & 4 & 4 & 4 & 4 & 4 & 3 & 4 & 4 & 3 & 34 & A \\
\hline $\begin{array}{l}\text { Bethancourt et al., } \\
2019 b^{(39)}\end{array}$ & 4 & 4 & 4 & 4 & 4 & 3 & 4 & 4 & 4 & 35 & A \\
\hline $\begin{array}{l}\text { Rodopaios et al., } \\
2019^{(5)}\end{array}$ & 4 & 4 & 4 & 4 & 4 & 3 & 4 & 4 & 3 & 34 & A \\
\hline $\begin{array}{l}\text { Karras et al., } \\
2019^{(46)}\end{array}$ & 4 & 4 & 4 & 4 & 4 & 3 & 4 & 4 & 3 & 34 & A \\
\hline $\begin{array}{l}\text { El-Sayed et al., } \\
2018^{(48)}\end{array}$ & 4 & 4 & 4 & 4 & 4 & 3 & 4 & 4 & 4 & 35 & $A$ \\
\hline $\begin{array}{l}\text { Makedou et al., } \\
2018^{(49)}\end{array}$ & 4 & 3 & 3 & 2 & 3 & 3 & 3 & 3 & 3 & 27 & B \\
\hline $\begin{array}{l}\text { Elshorbagy et al., } \\
2017^{(47)}\end{array}$ & 4 & 4 & 4 & 4 & 4 & 3 & 4 & 4 & 3 & 34 & A \\
\hline $\begin{array}{l}\text { Karras et al., } \\
2017^{(45)}\end{array}$ & 4 & 4 & 4 & 4 & 4 & 4 & 4 & 4 & 3 & 35 & $A$ \\
\hline Liali et al., 2015(44) & 4 & 3 & 4 & 3 & 3 & 3 & 3 & 3 & 3 & 29 & B \\
\hline $\begin{array}{l}\text { Papadaki et al., } \\
2011^{(42)}\end{array}$ & 4 & 4 & 4 & 4 & 4 & 3 & 4 & 4 & 3 & 34 & A \\
\hline $\begin{array}{l}\text { Chryssochoou } \\
\text { et al., 2010(34) }\end{array}$ & 4 & 3 & 4 & 4 & 4 & 3 & 4 & 4 & 3 & 33 & $A$ \\
\hline Sarri et al., 2009(38) & 4 & 4 & 4 & 4 & 4 & 3 & 4 & 4 & 3 & 34 & A \\
\hline $\begin{array}{l}\text { Papadaki et al., } \\
2007^{(43)}\end{array}$ & 4 & 4 & 4 & 4 & 4 & 3 & 4 & 4 & 3 & 34 & A \\
\hline Sarri et al., $2007^{(37)}$ & 4 & 4 & 4 & 4 & 4 & 3 & 4 & 4 & 3 & 34 & A \\
\hline Sarri et al., $2005^{(36)}$ & 4 & 4 & 4 & 4 & 4 & 3 & 4 & 4 & 3 & 34 & A \\
\hline Sarri et al., $2004^{(35)}$ & 3 & 4 & 4 & 4 & 4 & 3 & 4 & 4 & 3 & 33 & A \\
\hline Sarri et al., $2003^{(6)}$ & 4 & 4 & 4 & 4 & 4 & 3 & 4 & 4 & 3 & 34 & A \\
\hline $\begin{array}{l}\text { Basilakis et al., } \\
2002^{(41)}\end{array}$ & 4 & 3 & 4 & 3 & 4 & 3 & 4 & 4 & 3 & 32 & $A$ \\
\hline
\end{tabular}

